\title{
On implementing a spectral clustering controlled islanding algorithm in real power systems
}

DOI:

10.1109/PTC.2013.6652439

Link to publication record in Manchester Research Explorer

\section{Citation for published version (APA):}

Demetriou, P., Quiros-Tortos, J., Kyriakides, E., \& Terzija, V. (2013). On implementing a spectral clustering controlled islanding algorithm in real power systems. In 2013 IEEE Grenoble Conference PowerTech, POWERTECH 2013/IEEE Grenoble Conf. PowerTech, POWERTECH IEEE. https://doi.org/10.1109/PTC.2013.6652439

Published in:

2013 IEEE Grenoble Conference PowerTech, POWERTECH 2013|IEEE Grenoble Conf. PowerTech, POWERTECH

\section{Citing this paper}

Please note that where the full-text provided on Manchester Research Explorer is the Author Accepted Manuscript or Proof version this may differ from the final Published version. If citing, it is advised that you check and use the publisher's definitive version.

\section{General rights}

Copyright and moral rights for the publications made accessible in the Research Explorer are retained by the authors and/or other copyright owners and it is a condition of accessing publications that users recognise and abide by the legal requirements associated with these rights.

\section{Takedown policy}

If you believe that this document breaches copyright please refer to the University of Manchester's Takedown Procedures [http://man.ac.uk/04Y6Bo] or contact uml.scholarlycommunications@manchester.ac.uk providing relevant details, so we can investigate your claim.

\section{OPEN ACCESS}




\section{On Implementing a Spectral Clustering Controlled Islanding Algorithm in Real Power Systems}

\author{
Panayiotis Demetriou, Student Member, IEEE \\ University of Cyprus \\ Department of Electrical \& Computer Engineering \\ Nicosia, Cyprus \\ panayiotis_demetriou@ieee.org \\ Jairo Quirós-Tortós, Student Member, IEEE \\ The University of Manchester \\ School of Electrical \& Electronic Engineering \\ Manchester, UK \\ jairoquirostortos@ieee.org
}

\author{
Elias Kyriakides, Senior Member, IEEE \\ University of Cyprus \\ Department of Electrical \& Computer Engineering \\ Nicosia, Cyprus \\ elias@ucy.ac.cy \\ Vladimir Terzija, Senior Member, IEEE \\ The University of Manchester \\ School of Electrical \& Electronic Engineering \\ Manchester, UK \\ terzija@ieee.org
}

\begin{abstract}
Wide area blackouts can be caused by unexpected fault scenarios, in particular protection maloperation, or simultaneous low probability events, which as such might lead to e.g., un-damped electromechanical oscillations. A Spectral Clustering Controlled Islanding (SCCI) method to find a suitable islanding solution for preventing such events was previously proposed and tested using different IEEE test networks. The sole constraint applied to this solution was related to generator coherency. The method demonstrated promising results when it was implemented on these IEEE test networks. The SCCI method was later tested using a simplified Cypriot Network and the Polish Network. The results achieved when implementing the SCCI method on actual power systems highlight practical issues that were not previously considered and require to be addressed when using it. Therefore, this paper presents a robust SCCI method. An outlier problem which affects the quality of clustering and a problem with the computational efficiency of the algorithm (when dealing with systems larger than 500 nodes) are presented and analyzed in this paper. To improve the clustering quality, a more robust clustering algorithm, $k$-medoids, is used to cluster all nodes in the solution subspace and to find the islanding solution.
\end{abstract}

Index Terms--Controlled islanding, generator coherency, practical issues, robust clustering, spectral clustering.

\section{INTRODUCTION}

Recent blackouts in parts of Europe and America demonstrated the need for a systematic study and design of a comprehensive system control strategy. Intentional Control Islanding (ICI) is an efficient corrective measure for limiting the consequences of large disturbances which might eventually lead to a partial or complete blackout. When the loss of the power system integrity is inevitable, ICI can limit the occurrence and cost of blackouts by splitting the entire power system into smaller subsystems, also known as islands. The controlled islanding solution needs to determine the proper splitting points so that the formed islands can ensure a secure supply of the demand by stabilized local generators, without violating any transmission constraint.

ICI can be used to cope with different power system extremes, such as un-damped oscillations, voltage collapse, and cascading trips [1]. In order to create stable islands, the islanding solution must satisfy a large number of constraints such as load-generation balance, generator coherency, transmission line availability, voltage stability, and transient stability [1], [2]. In general, it would be too complicated to find a real-time solution that satisfies all these constraints, or even confirm if such a solution exists. In addition, the combinatorial explosion of the solution space that occurs for large power systems increases the complexity of solving the problem [3]. However, considering only a sub-set of these constraints, such as generator coherency and transmission line availability, allows a set of feasible candidate islanding solutions to be produced. This set of candidates can be coordinated with other corrective measures (e.g., transmission system reconfiguration (TSR), generation rescheduling (GR), and load shedding (LS)) to find a final islanding solution that satisfies all the constraints. This approximation reduces the complexity of the controlled islanding problem, especially when dealing with large-scale systems [1]-[5].

ICI is usually modeled as a combinatorial optimization problem with constraints. The two main types of its objective function are minimal power-flow disruption and minimal power imbalance within islands, whilst the constraints are coherent generator groups [3]. The power-flow disruption can be expressed by the arithmetical sum of active power in each disconnected transmission line. Techniques for minimal power-flow disruption minimize the change of the power flow pattern within the system following system splitting [6], [7]. On the other hand, the power imbalance can be expressed by the algebraic sum of active power on each disconnected transmission line (considering the direction of power flow). 
Techniques for finding islanding solutions with minimal power imbalance minimize the power imbalance within the formed islands to reduce the amount of load that must be shed and the generation to be rescheduled after system splitting [1], [4], [8].

To solve the combinatorial optimization problem with minimal power imbalance, several methods have been proposed in the literature. In [4] and [5], a two-phase Ordered Binary Decision Diagram (OBDD) method based on a simplified graph and Breadth First Search (BFS)/Depth First Search (DFS) algorithms are presented to find power-balanced islands containing coherent generator groups. According to [4], the combinatorial optimization problem with minimal power imbalance is NP-hard. In other words, it is very difficult to find a solution for a large-scale system within polynomial time. To overcome this problem, the original power system must be simplified to less than 40 nodes [3].

Spectral Clustering (SC) and multi-level kernel $k$-means have been used to find the islanding solution with the minimal power-flow disruption [6]. This technique exhibits excellent computational efficiency. Nevertheless, it requires the computation of the eigenvalues and eigenvectors associated with the graph employed to model the power system. In addition, the fact that the technique does not consider the dynamic (coherent groups of generators) and static (unavailable transmission lines) constraints, may lead to two unacceptable solutions for system splitting: (i) a simple separation of one node from the rest of the graph and (ii) a solution where the stability of islands formed cannot be guaranteed.

Identifying this disadvantage in the existing SC solution, a novel two-step Spectral Clustering Controlled Islanding (SCCI) algorithm, ensuring only the dynamic constraint, is proposed in [3]. The method was tested and validated using three different IEEE test systems. The results in this paper were promising and demonstrated the effectiveness of the SCCI method in networks with up to 118 buses. Nevertheless, the method was later tested in various real networks, including small systems and very large-scale networks. The results achieved, when implementing the SCCI algorithm on real power systems, highlighted practical issues previously not considered that require to be addressed when using the existing SCCI method. Therefore, the previously proposed SCCI algorithm is improved in this paper and a Robust SCCI (RSCCI) is presented. The existing SCCI and the RSCCI methods are tested in this paper using a simplified Cypriot Network [15] and the Polish Network available in MatPower [9]. The simplified Cypriot network resembles the actual Cypriot network [15]. The Polish Network available in MatPower represents the Polish 400, 220 and $110 \mathrm{kV}$ networks during winter 2007-08 evening peak conditions and includes some equivalents of the German, Czech and Slovak networks.

In particular, the existing SCCI does not include constraints to unavailable transmission lines. Thus, transformers and lines without synchro-check relays might eventually be included in the islanding solution. Synchrocheck relays are essential during the synchronization of islands during the restoration process. Furthermore, the existing SCCI is also sensitive to outliers and computationally expensive for large scale (over 500 nodes) power systems. An outlier is an observation that is numerically distant from the rest of the data [16]. Therefore, this paper presents possible solutions for when such problems occur. An outlier detection algorithm, to determine the optimal splitting strategy in strong connected networks and a constraint to exclude unavailable transmission lines from the splitting strategy are detailed in this paper to determine the optimal splitting strategy in large scale power systems. To reduce the computation of the existing SCCI, a reduced area i.e. reduced searching space, which concentrates potential splitting strategies, is proposed prior the application of the clustering technique.

\section{SPECTRAL CluStering CONTROLLED ISLANDING ALGORITHM}

In this Section, the graph theory fundamentals are given. The ICI problem is then defined as a constrained combinatorial optimization problem and a graph-cut problem.

\section{A. Graph theory fundamentals}

In graph theory, an undirected graph-model $\mathbf{G}\left(\mathbf{V}, \mathbf{V}_{\mathbf{G}}, \mathbf{E}, \mathbf{W}\right)$ can be used to describe an $m$-generator and $n$-bus power system. In this graph-model, the node set $\mathbf{V}=\left\{v_{1}, \ldots, v_{n}\right\}$ denotes the buses while the edge set $\mathbf{E}$ with elements $e_{i j}(i, j=1, \ldots, n)$ denotes the transmission lines. $\mathbf{V}_{\mathbf{G}}$ is a subset of the node set $\mathbf{V}$ that contains only those buses with generators directly connected to them. The set $\mathbf{W}$, with elements $w_{i j}(i, j=1, \ldots, n)$ is a set of edge weights.

Bisection of the graph $\mathbf{G}$ means splitting the graph $\mathbf{G}$ into two sub-graphs $\mathbf{G}_{\mathbf{1}}\left(\mathbf{V}_{\mathbf{1}}, \mathbf{V}_{\mathbf{G 1}}, \mathbf{E}_{\mathbf{1}}, \mathbf{W}_{\mathbf{1}}\right)$ and $\mathbf{G}_{\mathbf{2}}\left(\mathbf{V}_{\mathbf{2}}, \mathbf{V}_{\mathbf{G} \mathbf{2}}, \mathbf{E}_{\mathbf{2}}, \mathbf{W}_{\mathbf{2}}\right)$ by removing the edges connecting these two sub-graphs [10]. Here, $\mathbf{V}_{\mathbf{1}}$ and $\mathbf{V}_{\mathbf{2}}$ are disjoint subsets of $\mathbf{V}\left(\mathbf{V}_{\mathbf{1}} \cup \mathbf{V}_{\mathbf{2}}=\varnothing\right.$ and $\left.\mathbf{V}_{\mathbf{1}} \cap \mathbf{V}_{\mathbf{2}}=\mathbf{V}\right)$. $\mathbf{V}_{\mathbf{G} \mathbf{1}}$ and $\mathbf{V}_{\mathbf{G} \mathbf{2}}$ are two disjoint subsets of $\mathbf{V}_{\mathbf{G}}$ which are also subsets of $\mathbf{V}_{\mathbf{1}}$ and $\mathbf{V}_{\mathbf{2}}$, respectively. The set of edges removed to separate these sub-graphs is called the cutset [3]. The sum of the weights of the edges within this cutset is called the cut, which is defined as in equation (1).

$$
\operatorname{cut}\left(\mathbf{V}_{\mathbf{1}}, \mathbf{V}_{\mathbf{2}}\right)=\sum_{i \in \mathbf{V}_{\mathbf{1}}, j \in \mathbf{V}_{\mathbf{2}}} w_{i j}
$$

The ICI problem is then converted into the problem of finding the cutset that bisects a graph with minimal cut [10]. The un-normalized SC method, which clusters the nodes into two sub-sets based on the Laplacian matrix $\mathbf{L}$, can be used to solve this problem. For a graph $\mathbf{G}, \mathbf{L}$ is defined as:

$$
\mathbf{L}=\mathbf{D}-\mathbf{W}
$$

where $\mathbf{D}=\operatorname{diag}\left(d_{i}\right), i=1, \ldots, n$, is a diagonal degree matrix that contains the diagonal elements $d_{i}$ which is equal to the total weight of the edges connected to node $i$. The weighted adjacency matrix $\mathbf{W}=\left(w_{i j}\right), i, j=1, \ldots, n$, is a set of edge weights.

For the bisection case, the un-normalized SC algorithm can be described by the following steps [10]:

1. Compute the first two eigenvectors $\boldsymbol{\theta}_{\mathbf{1}}, \boldsymbol{\theta}_{\mathbf{2}}$, associated with the two smallest eigenvalues, of $\mathbf{L}$. 
2. Let $\mathbf{J} \in \mathbf{R}^{n \times 2}$ be the matrix containing the vectors $\boldsymbol{\theta}_{1}, \boldsymbol{\theta}_{2}$ as columns. Let $\mathbf{y}_{i} \in \mathbf{R}^{2}$ be the vector corresponding to the $i$-th row of $\mathbf{J}$.

3. Cluster the nodes $\mathbf{y}_{i} \in \mathbf{R}^{2}$ into the clusters $\mathbf{C}_{\mathbf{1}}, \mathbf{C}_{\mathbf{2}}$ using a clustering algorithm, such as the $k$-means algorithm.

\section{B. Intentional Controlled Islanding}

The ICI problem, for solving the minimal power-flow disruption, can be represented as a constrained combinatorial optimization, with the objective function given in (3).

$$
\min _{\mathbf{V}_{1}, \mathbf{V}_{2} \subset \mathbf{V}}\left(\sum_{i \in \mathbf{V}_{1}, j \in \mathbf{V}_{2}}\left|P_{i j}\right|\right) \text { s.t. } \mathbf{V}_{\mathbf{G} 1} \subset \mathbf{V}_{\mathbf{1}}, \mathbf{V}_{\mathbf{G} 2} \subset \mathbf{V}_{2}, \mathbf{E}_{\mathbf{C}} \subset \mathbf{E}
$$

where $\left|P_{i j}\right|$ denotes the absolute value of the active power exchange on the transmission line between node $i$ and $j . \mathbf{E}_{\mathbf{C}}$ is an edge subset that contains all the branches that cannot be disconnected.

The use of minimal power-flow disruption as the objective function minimizes the sum of the absolute values of the active power exchange between islands, also called absolute power exchange. This property improves the transient stability of the formed islands, reduces the possibility of overloading the transmission lines within the newly created islands and makes islands resynchronization easier [1], [14]. In contrast, the use of minimal power imbalance as the objective function creates islands with good load-generation balance. An island with a negative stability margin and a good load-generation balance will collapse [14]. However, an island with a positive stability margin and a poor load-generation balance can be stabilized through load shedding. Therefore, considering that the primary concern to ensure stability after system splitting should be transient stability rather than load-generation balance, the minimal power-flow disruption is used in this paper. The constraints applied when satisfying this objective function deal with coherent generator groups and line availability.

\section{Constrained Spectral Clustering Algorithm}

As discussed in Section I, the neglect of generator coherency often leads to producing unstable islands and direct application of SC without any constraint also tends to produce isolated load nodes. This section introduces the procedure for obtaining a set of feasible solutions by constrained SC. The flowchart of a constrained SC algorithm for bisection is shown in Fig. 1.

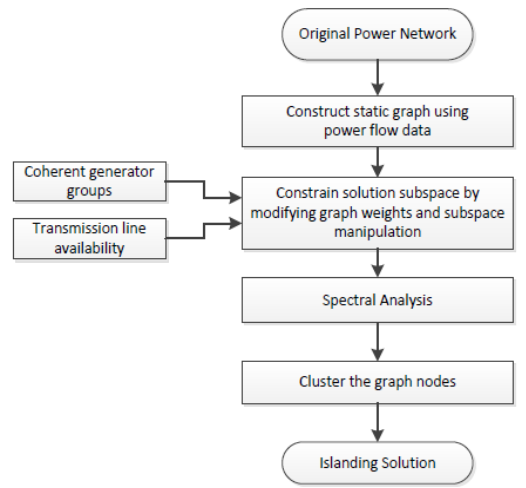

Figure 1. Flowchart of the CSC algorithm for the bisection case
When subjected to large disturbances, some synchronous machines tend to swing together. Such coherent machines can be grouped into coherent groups. Many real-time algorithms have been designed to determine these coherent groups of generators [11]. Therefore, since Wide Area Monitoring Systems (WAMS) accompanied by telecommunication infrastructure [17]-[19] are still under development and implementation in different utilities worldwide, this paper considers that it will be possible to determine these coherent groups of generators using WAMS. After successfully determining the groups of coherent generators, this information can then be used as an input variable for the actual SCCI and RSCCI methods.

\section{1) Constraints in the SCCI}

A pair-wise constraint can be used to describe the relationship between two nodes of the graph $\mathbf{G}$. This consists of a must-link or cannot-link constraint between any two nodes [12]:

Must-link constraint: node $i$ and node $j$ must be linked.

Cannot-link constraint: node $i$ and node $j$ cannot be linked.

By defining the must-link and cannot-link constraints, the constraints due to coherent generator groups and transmission line availability can be converted into a set of pair-wise constraints. With these pair-wise constraints, the ICI problem is converted into a constrained clustering problem which can be solved using the CSC. In order to incorporate these constraints efficiently, the modifying graph weights is firstly used to handle the pair-wise constraints about transmission line availability, and the subspace manipulation is then used to handle the pair-wise constraints about generator coherency.

\section{a) Modifying graph weights}

The RSCCI method applies constraints to unavailable transmission lines. When a transmission line is unavailable to trip, the weight factor associated with the edge is changed as described in (4).

$$
\text { If } e_{i j} \in \mathbf{E}_{\mathbf{C}}, \text { set } w_{i j}=w_{j i}=\max (\mathbf{W})
$$

\section{b) Subspace manipulation}

The constraints generated from generator groups can be incorporated by modifying the solution subspace using a projection matrix $\mathbf{U}$. Assuming that the first $m_{l}$ nodes belong to the cluster $\mathbf{C}_{\mathbf{1}}$ and the next $m_{2}$ nodes belong to the cluster $\mathbf{C}_{\mathbf{2}}$, the projection matrix can be defined as [3]:

$$
\mathbf{U}=\left[\begin{array}{ccc}
1_{m_{1}} & 1_{m_{1}} & 0_{m_{1} x(n-m)} \\
1_{m_{2}} & -1_{m_{2}} & 0_{m_{2} x(n-m)} \\
1_{n-m} & 0_{n-m} & \mathrm{I}_{(n-m) x(n-m)}
\end{array}\right]
$$

where $\mathbf{I}$ is the identity matrix, $\mathbf{1}$ is the all-ones column vector, and $\mathbf{0}$ is the zero column vector.

By this way, the solution subspace is constrained from an $n$-dimension space to a $(n-m+2)$ dimension subspace, where $\left(m=m_{1}+m_{2}\right)$. In this subspace, the nodes with must-link in the 
$n$-dimension space are merged together, while the nodes with cannot-link in the $n$-dimension space are separated, to satisfy the pair-wise constraints. With the introduction of the projection matrix $\mathbf{U}$, constrained $\mathrm{SC}$ can be applied to the static graph $\mathbf{G}_{\mathbf{S}}$ to find the cutset with minimal power-flow disruption that satisfies the generator grouping constraints.

\section{2) Spectral Analysis}

The SCCI algorithm uses a static graph $\mathbf{G}_{\mathbf{S}}\left(\mathbf{V}, \mathbf{E}_{\mathbf{S}}, \mathbf{W}_{\mathbf{S}}\right)$ which is constructed using power flow data. This graph contains every node of the power system and its edge weights are defined as the absolute value of the active power exchange between nodes $i$ and $j,\left|P_{i j}\right|$. The Laplacian matrix $\mathbf{L}_{\mathbf{S}}$ of the static graph $\mathbf{G}_{\mathbf{S}}$ can be expressed as [3]:

$$
\left[\mathrm{L}_{\mathrm{S}}\right]_{i j}= \begin{cases}\frac{\left|P_{i j}\right|+\left|P_{j i}\right|}{2}=-\left|V_{i}\right|\left|V_{j}\right| B_{i j} \sin \left(\delta_{i}-\delta_{j}\right) & \text { if } i \neq j \\ -\sum_{l=1, l \neq i}^{n}\left[\mathrm{~L}_{\mathrm{S}}\right]_{i l} & \text { if } i=j\end{cases}
$$

where $B_{i j}$ is the imaginary entry of the network admittance matrix.

The nodes are then grouped using constrained SC to solve the optimization problem with the minimal power-flow disruption. In summary, the existing SCCI algorithm can be executed as follows:

1. Construct a static graph $\mathbf{G}_{\mathbf{S}}$ of all nodes with the edge weights defined as $\left(\left|P_{i j}\right|+\left|P_{j i}\right|\right) / 2$.

2. Construct the projection matrix $\mathbf{U}$ based on the generator grouping results.

3. Compute the first two eigenvectors $\boldsymbol{\theta}_{1}, \boldsymbol{\theta}_{2}$ of the generalized Eigen-problem $\mathbf{U}^{\mathrm{T}} \mathbf{L}_{\mathbf{S}} \mathbf{U} \boldsymbol{\theta}=\lambda \mathbf{U}^{\mathrm{T}} \mathbf{U} \boldsymbol{\theta}$.

4. Let $\mathbf{J} \in \mathbf{R}^{n \times 2}$ be the matrix containing the vectors $\mathbf{U} \boldsymbol{\theta}_{\mathbf{1}}$, $\mathbf{U} \boldsymbol{\theta}_{2}$ as columns. Let $\mathbf{y}_{i} \in \mathbf{R}^{2}$ be the vector corresponding to the $i$-th row of $\mathbf{J}$.

5. Cluster the nodes $\mathbf{y}_{i} \in \mathbf{R}^{2}$ into the clusters $\mathbf{V}_{\mathbf{1}}, \mathbf{V}_{\mathbf{2}}$ using the k-means algorithm.

6. Select $\mathbf{V}_{1}$ or $\mathbf{V}_{2}$ as the node set of a new static graph and return to 1$)$.

\section{Outlier detection and robust clustering}

As mentioned in the previous fifth point, after running the spectral analysis of the graph, a proper clustering algorithm should be selected to cluster the nodes of the graph based on the selected eigenvectors. There are many clustering algorithms that could be used. While $k$-means, the clustering algorithm used in the existing SCCI method, is the traditional and default algorithm in the literature as it is simple and fast, it is very sensitive to outliers [12]. The k-means attempts to minimize a squared error function described as follows:

$$
\mathbf{J}=\sum_{j=1}^{k} \sum_{i=1}^{n}\left\|x_{i}^{(j)}-c_{j}\right\|^{2}
$$

where $\left\|x_{i}^{(j)}-c_{j}\right\|^{2}$ is a chosen distance measure between a data-point $x_{i}^{(j)}$ and the cluster center (centroids) $c_{j}$, the mean value of the data-points in the cluster $j$.

When applying spectral analysis to solve the generalized eigenvalue problem, it is possible for graph nodes with very small weights and/or small degrees (e.g. a one-degree node with a very small weight) to produce eigenvectors that are outliers. Since the SC algorithm clusters nodes based on the eigenvectors of the graph, these outliers will affect the quality of the clustering.

There are two ways to handle this outlier problem. One is detecting the outliers through outlier detection algorithms and eliminating it before clustering. Another way is using a robust clustering algorithm which is not sensitive to outliers. In this paper, a more robust clustering algorithm, $k$-medoids, to cluster the nodes in the solution subspace is implemented. Thus, a RSCCI method is obtained from the existing SCCI method.

Instead of taking the mean value of data-points in a cluster as the cluster center, $k$-medoids chooses the most centrally located data-point in a cluster (medoids) as the cluster center. It is more robust to noise and outliers compared to $k$-means because it minimizes a sum of dissimilarities instead of a sum of squared distances between data-points [13].

\section{E. Reduced Search Space}

Direct application of the existing SCCI on large scale power system is not possible since the computation time is not acceptable for real-time application. Therefore, it is vital to reduce the search space and apply the RSCCI within the reduced search space. Even though this paper does not present such solution, the authors are working on potential solutions like this.

\section{SimULATION RESUlTS}

This Section presents the simulation results for two realcase systems: the Cypriot network [15] and the Polish Network available in MatPower [9].

\section{A. Cypriot network}

The simplified Cypriot network resembling the actual network is firstly used to demonstrate the problems caused by outliers. This test system is also used to constrain the solution when unavailable transmission lines are detected in the system. This equivalent system has 5 synchronous generators, 48 buses and 84 branches. The total system production during the off-peak winter load time is $544.32 \mathrm{MW}$ while the total consumption $543 \mathrm{MW}$. It is assumed that this system will be split into two islands. Employing real-time solutions [11], the following two coherent groups of generators are assumed $\{1,15,48\}$ and $\{25,27\}$. This information is used to build the matrix $\mathbf{U}$, which contains the must-link and cannot-link constraints. It is also assumed that the following lines cannot be included in the splitting strategy: $\{13-43,42-43$, and 22$24\}$. Thus, the weight factor associated with these edges is changed applying (4). Implementing the existing SCCI algorithm [3], the solution obtained does not satisfy the 
coherency constraint. As shown in Fig. 2, this solution does not separate the non-coherent groups of machines. Thus, transient instability within the islands might lead to a blackout. In addition, the solution obtained separates load nodes without a generator. An island without generation will unavoidably collapse.

The incorrect solution obtained by the existing SCCI is due to the presence of outliers. As shown in Fig. 3, which plots the value of the eigenvector associated with the second smallest eigenvalue, there are four outliers (data-points 35, 39, $43,46)$. As noticed, using the k-means, the solution obtained splits three of these outliers from the rest of the data. Thus, the existing SCCI incorrectly splits the power system. As previously mentioned, this incorrect splitting might eventually lead to a complete blackout.

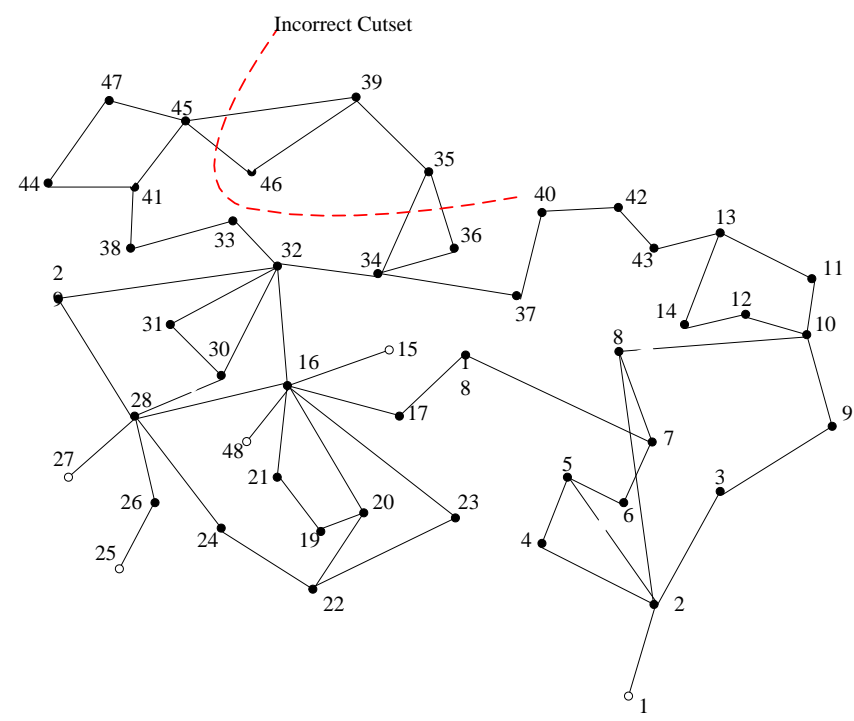

Figure 2. Cypriot Network split into two islands: Incorrect solution determined by existing SCCI algorithm

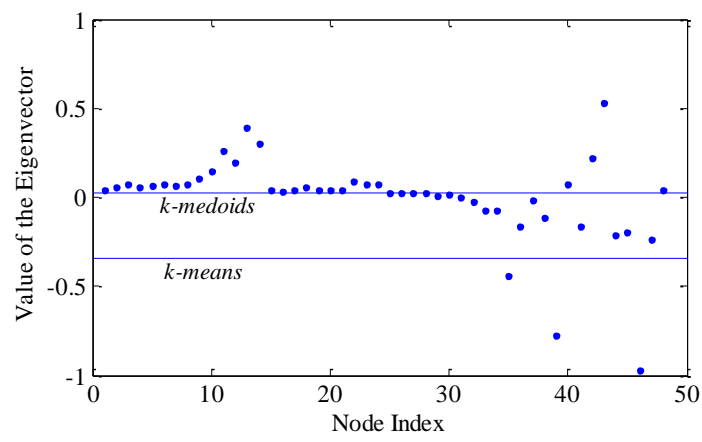

Figure 3. Value of the Eigenvector associated with the second smallest eigenvalue

By implementing the $k$-medoids algorithm, the solution with the RSCCI solves the problem of outliers and detects the new solution. As it can be noticed in Fig. 4, this new solutions splits the power system into two islands. As expected this solution minimizes the power-flow disruption, satisfying the constraint of grouping the coherent groups of generators. As the RSCCI method can also cope with unavailable lines in the system, the obtained solution excludes the lines 42-43 and 22-
24 which would have been included in the optimal solution instead of lines 37-40 and 24-28, respectively.

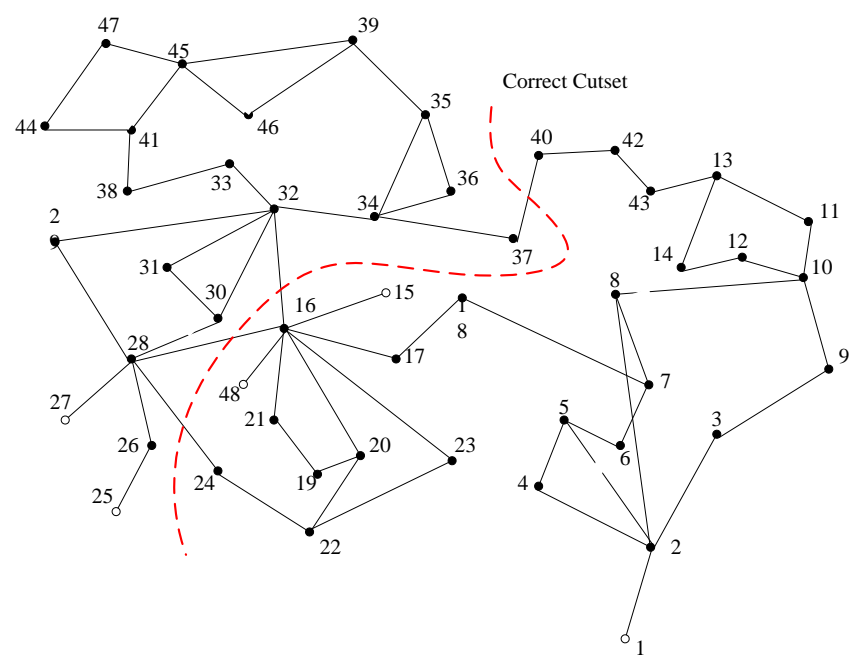

Figure 4. Cypriot Network split into two islands: Correct solution using the RSCCI method

\section{B. Polish network}

The Polish network, available in MatPower [9], is further used to test both the SCCI and the RSCCI methods. This test system contains 3375 buses. Application of the existing SCCI demonstrates that the methods require more than $45 \mathrm{~s}$ to determine the controlled islanding solution for minimal power-flow disruption. The complexity of the SC algorithm is due to the computation of the eigenvalues associated with the Eigen-problem described II-A. The complexity is increased in CSC methods by introducing the projection matrix $\mathbf{U}$ and determining the constrained solution for the controlled islanding. Fig. 5 shows a direct comparison of the time required by both the existing SCCI and the RSCCI methods.

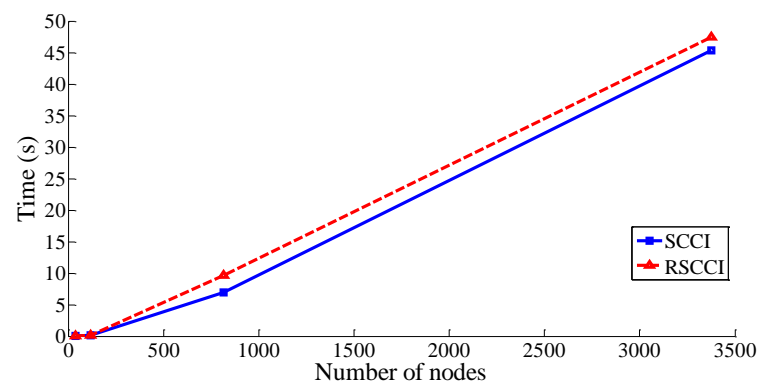

Figure 5. Performance of the SCCI and the RSCCI methods in function of the number of nodes

As noticed in Fig. 5, both methods have similar computation time. While the RSCCI is slightly slower (requires less than $5 \%$ of extra time respect to the existing SCCI in the analyzed cases), it will determine the optimal solution despite the presence of outliers. As it can be noticed, direct application of the existing SCCI, or the RSCCI, method in networks with more than 500 nodes is not acceptable for real-time application. From Fig. 5, it can be concluded that both the SCCI and the RSCCI are effective when the network, or a reduced set of nodes representing a reduced search space, is equal to 300 nodes, for which cases, both methods are 
expected to solve the problem in less than 2 seconds. However, as the RSCCI solves the problem of outliers and excludes certain branches from the splitting strategy, the RSCCI is better than the existing SCCI method.

\section{CONCLUSION AND FUTURE WORK}

An existing Spectral Clustering Controlled Islanding (SCCI) method [3] was further validated and improved using cases of real transmission networks of Cyprus and Poland. The application of this method on these networks highlighted some critical practical issues that have not been previously considered and that require to be addressed before implementing the method in real networks.

In particular, the existing method is sensitive to outliers and does not constrain certain branches to be excluded from the splitting strategy. Therefore, this paper implemented a robust clustering algorithm, $k$-medoids, to solve the problem of outliers. As a consequence, a new Robust SCCI (RSCCI) method was developed. To constrain branches to be excluded from the islanding solution, the weight factors associated with the edges to be excluded are changed to the maximum weight value in the weighted adjacency matrix. Using this constraint, it is ensured that unavailable branches are not included in the splitting strategy.

The existing SCCI and the proposed RSCCI methods were also tested on large-scale networks and its computation time was found to be expensive. The size of the network versus the computation time was studied and it was determined that the existing SCCI and the RSCCI are suitable for real-time applications, when the number of nodes is smaller than 300 nodes. Since current power systems contain several nodes, it is proposed to reduce the search space to a common and potential area, where various splitting strategies can be determined.

The determination of this reduced search space will improve the computation and the effectiveness of both methods, making these suitable for real-time applications.

\section{REFERENCES}

[1] K. Sun, D.Z. Zheng and Q. Lu, "Splitting strategies for islanding operation of large-scale power systems using OBDD-based methods," IEEE Transactions on Power Systems, vol. 18, no. 2, pp. 912-923, May 2003.

[2] H. You, V. Vittal and X. Wang, "Slow coherency-based islanding," IEEE Transactions on Power Systems, vol. 19, no.1, pp. 483-491, Feb. 2004.

[3] L. Ding, F.M. Gonzalez-Longatt, P. Wall and V. Terzija, "Two-step spectral clustering controlled islanding algorithm," IEEE Transactions on Power Systems, vol. 28, no. 1, pp. 75-84, Feb. 2013.

[4] Q. Zhao, K. Sun, D.Z. Zheng, J. Ma and Q. Lu, "A study of system splitting strategies for island operation of power system: A two-phase method based on OBDDs," IEEE Transactions on Power Systems, vol. 18, no. 4, pp. 1556-1565, Nov. 2003.

[5] X. Wang, "Slow coherency grouping based islanding using minimal cutsets and generator coherency index tracing using the continuation method," Ph.D., Iowa State University, Ames, 2005.

[6] H. Li, G.W. Rosenwald, J. Jung and C. Liu, "Strategic power infrastructure defense," Proceedings of the IEEE, vol. 93, no. 5, pp. 918- 933, May 2005.

[7] B. Yang, V. Vittal, G.T. Heydt and A. Sen, "A novel slow coherency based graph theoretic islanding strategy," Presented at Power Engineering Society General Meeting, Jun. 2007.

[8] W. Liu, L. Liu and D.A. Cartes, "Slow coherency and angle modulated particle swarm optimization based islanding of large scale power systems," Presented at Neural Networks, International Joint Conference, IJCNN, Aug. 2007.

[9] R.D. Zimmerman, C.E. Murillo-Sánchez and R.J. Thomas, "MATPOWER: Steady-state operations, planning, and analysis tools for power systems research and education," IEEE Transactions on Power Systems, vol. 26, no. 1, pp. 12-19, Feb. 2011.

[10] J.A. Bondy and U.S.R. Murty, "Graph Theory," 2nd Ed:Springer, 2008.

[11] M. Jonsson, M. Begovic and J. Daalder, "A new method suitable for real-time generator coherency determination," IEEE Transactions on Power Systems, vol. 19, no. 3, pp. 1473-1482, Aug. 2004.

[12] T.D. Bie, J. Suykens, and B.D. Moor, "Learning from general label constraints," inIAPR International Workshop on Statistical Pattern Recognition, Lisbon, 2004.

[13] D. Cao and B. Yang, "An improved k-medoids clustering algorithm," in The 2nd International Conference on Computer and Automation Engineering, Feb. 2010.

[14] V.E. Henner, "A network separation scheme for emergency control," Int. J. Elect. Power Energy Syst., vol. 2, no. 2, pp. 109-114, 1980.

[15] V.G. Koutsoloukas, "Modelling of Cypriot power system on PSS/SinCal, study of disturbances, and influence of wind penetration," Dissertation, National Technical University, 2007 (in Greek).

[16] S. Theodoridis, K. Koutroumba, "Pattern Recognition," 4th Ed:American Press, 2008.

[17] V. Terzija, G. Valverde, D. Cai, P. Regulski, V. Madani, J. Fitch, S. Skok, M. Begovic, A. Phadke, "Wide Area Monitoring, Protection and Control of Future Electric Power Networks," Proceedings of IEEE, vol. 99, no. 1, pp 80-93, Jan., 2011.

[18] V. Terzija, D. Cai, A. Vaccaro, J. Fitch, "Architecture of wide area monitoring systems and their communication requirements", Paris Cigre Session, 2010

[19] S. Chakrabarti, E. Kyriakides, B. Tianshu, D. Cai and V. Terzija, "Measurements get together", IEEE Power and Energy Magazine, vol. 7, no. 1, pp 41-49, Jan.- Feb., 2009. 